\title{
Critical Temperature Curve in BEC-BCS Crossover
}

\author{
Evgeni Burovski, ${ }^{1}$ Evgeny Kozik, ${ }^{2,3}$ Nikolay Prokof'ev, ${ }^{2,3,4}$ Boris Svistunov, ${ }^{2,4}$ and Matthias Troyer ${ }^{3}$ \\ ${ }^{1}$ Laboratoire de Physique Théorique et Modèles Statistiques, Université Paris-Sud, 91405 Orsay Cedex, France \\ ${ }^{2}$ Department of Physics, University of Massachusetts, Amherst, Massachusetts 01003, USA \\ ${ }^{3}$ Theoretische Physik, ETH Zurich, 8093 Zurich, Switzerland \\ ${ }^{4}$ Russian Research Center "Kurchatov Institute," 123182 Moscow, Russia
}

(Received 20 May 2008; published 27 August 2008)

\begin{abstract}
The strongly correlated regime of the crossover from Bardeen-Cooper-Schrieffer pairing to BoseEinstein condensation can be realized by diluting a system of two-component fermions with a short-range attractive interaction. We investigate this system via a novel continuous-space-time diagrammatic determinant Monte Carlo method and determine the universal curve $T_{c} / \varepsilon_{F}$ for the transition temperature between the normal and the superfluid states as a function of the scattering length with the maximum on the Bose-Einstein condensation side. At unitarity, we confirm that $T_{c} / \varepsilon_{F}=0.152(7)$.
\end{abstract}

PACS numbers: 05.30.Jp, 03.75.Ss, 05.10.Ln, 71.10.Fd

In the area of ultracold gases, the problem of the crossover between the Bardeen-Cooper-Schrieffer pairing and the Bose-Einstein condensation (BEC) of composite molecules (the so-called BCS-BEC crossover) has recently received a lot of theoretical and experimental attention [1]. A dilute two-component Fermi gas, where the interparticle distance is much larger than the interaction range, features a remarkable universality at low temperatures. Since the interaction is completely described by the $s$-wave scattering length $a$, the only physically relevant coupling parameter is $\kappa=1 / k_{F} a$, where $k_{F}$ is the Fermi momentum. One thus obtains a unified and universal description of systems as diverse as ultracold fermionic gases in magnetic or optical traps [1], fermions in optical lattices, inner crusts of neutron stars [2,3], and, plausibly, excitonic condensates [4].

In the limit $\kappa \rightarrow-\infty$, the Fermi gas is described by the BCS theory, while for $\kappa \rightarrow+\infty$ the fermions pair into compact bosonic molecules which then form a BEC state below the critical temperature. Separating these extreme states is a strongly correlated regime which features the socalled unitary point $\kappa=0$. At unitarity, the scattering length is infinite and the interaction thus drops out of the relations between different thermodynamic potentials making these relations formally identical to those of a noninteracting Fermi gas [5]. On the experimental side, using the technique of a (wide) Feshbach resonance in a system of cold atoms, one can traverse the whole range of parameter $\kappa$ from the BEC to the BCS limit [1].

Despite considerable recent investigation, the quantitative description of the BEC-BCS crossover is far from being complete, even for the simplest case of the equal mixture of two components. Because of the strongly correlated nature of the problem, analytical mean-field-type calculations (e.g., [6-8]) unavoidably involve approximations, the accuracy of which is difficult to access unless the exact result is known. Renormalization group treatments can be carried out as expansions in either $\epsilon=4-d[9]$ or
$1 / N_{F}$ (where $N_{F}$ is the number of fermion species) [10,11], but the applicability of these calculations to the physically relevant case of $d=3$ and $N_{F}=2$ is not known a priori.

Numerical studies of fermionic systems are computationally demanding and further complicated by the need to study the limit of small densities to access the universal regime. Some numerical techniques avoid the fermionic sign problem with a help of uncontrollable approximations. The restricted path-integral Monte Carlo method [12] relies on a variational ansatz for the nodes of the density matrix. In the dynamical mean-field theory approach of Ref. [13] the physics of extended paired states is reduced to that of a single site coupled to the selfconsistently defined environment. Fortunately, the unpolarized Fermi gas with contact attraction is an exceptional case which can be addressed by sign-problem-free determinant methods without uncontrollable systematic errors [14-16]. Moreover, the determinant diagrammatic MC approach (DDMC) for lattice fermions [15] is completely free of any systematic error. In simulations of the negative- $U$ Hubbard model [14] with an appropriate extrapolation to zero filling at the unitary point, we previously obtained accurate results for the critical temperature of the superfluid transition, $T_{c} / \varepsilon_{F}=0.152(7)$, in the units of the Fermi energy $\varepsilon_{F}$. This result, however, did not agree with the estimate obtained by Ref. [16] from a visual inspection of the caloric curve, using the standard auxiliary field approach [17].

To efficiently study the critical temperature curve away from the unitarity point and to verify that the final results are model independent - thereby also resolving the controversy on $T_{c} / \varepsilon_{F}$ at unitarity-we develop a DDMC technique for continuous space and time. We can now efficiently simulate models with a simple parabolic dispersion relation and have completely eliminated lattice corrections. In this Letter, we first discuss the new scheme and how to obtain an independent systematic-error-free value for $T_{c} / \varepsilon_{F}$ at unitarity. We are able to reach densities almost 
20 times smaller than those typically accessible with the auxiliary field determinant method [16], This allows us to perform a reliable extrapolation to the universal limit yielding $T_{c} / \varepsilon_{F}=0.152(7)$, in perfect agreement with our previous value [14]. Next, we explore the critical temperature at finite values of $1 / k_{F} a$. Our results, shown in Fig. 1, fix the general shape of the universal curve $T_{c} / \varepsilon_{F}$ versus $1 / k_{F} a$. The main feature is a substantial maximum of $T_{c} / \varepsilon_{F}$ on the BEC side of the crossover.

Our specific model is described by the Hamiltonian

$$
\begin{aligned}
H= & \sum_{\sigma=\uparrow, \downarrow} \int d \mathbf{x} \Psi_{\sigma}^{\dagger}(\mathbf{x})(\hat{K}-\mu) \Psi_{\sigma}(\mathbf{x}) \\
& +U \int d \mathbf{x} \Psi_{\uparrow}^{\dagger}(\mathbf{x}) \Psi_{\downarrow}^{\dagger}(\mathbf{x}) \Psi_{\downarrow}(\mathbf{x}) \Psi_{\uparrow}(\mathbf{x}),
\end{aligned}
$$

where $\Psi_{\sigma}(\mathbf{x})$ is the fermion field operator $(\sigma=\uparrow, \downarrow)$, $\mathbf{x}$ is a continuous three-dimensional coordinate, $\mu$ is the chemical potential, $U<0$ is the contact interaction strength, and $\hat{K}$ is the kinetic energy operator, $\hat{K} e^{i \mathbf{k} \mathbf{x}}=\varepsilon_{\mathbf{k}} e^{i \mathbf{k} \mathbf{x}}$, with $\varepsilon_{\mathbf{k}}$ being the single-particle dispersion.

The scattering length $a$ is given by the sum of the vacuum ladder diagrams [18] leading to $(\hbar=1)$

$$
\frac{m}{4 \pi a}=U^{-1}+\int \frac{d \mathbf{k}}{(2 \pi)^{3}} \frac{1}{2 \varepsilon_{\mathbf{k}}},
$$

where $m$ is the fermion mass. For the continuous space model with $\varepsilon_{\mathbf{k}}=k^{2} / 2 m$ an ultraviolet regularization of Eq. (2) is required. Keeping in mind comparison with Ref. [16], where the parabolic dispersion with an ultraviolet cutoff was used, we introduce a microscopic length

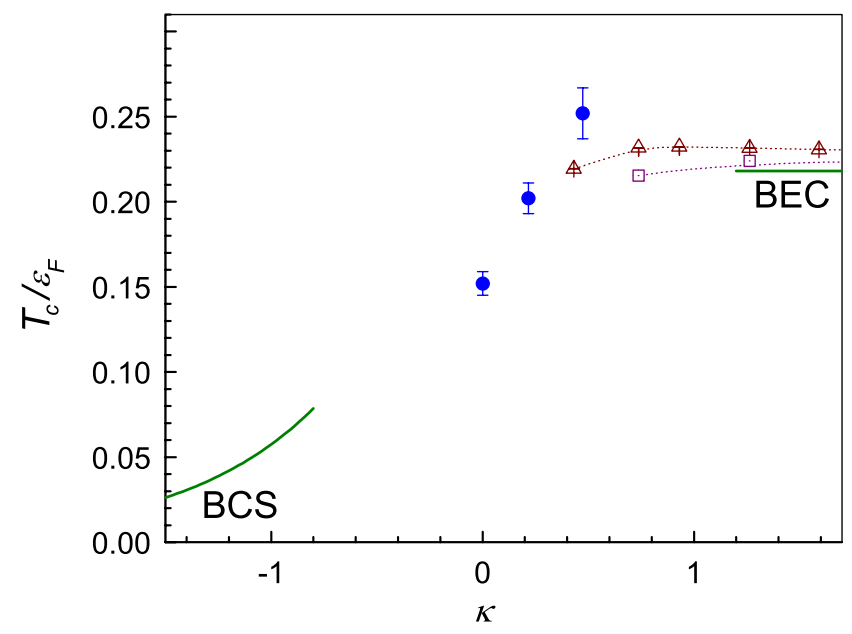

FIG. 1 (color online). The universal results for the critical temperature in the units of the Fermi energy plotted versus $\kappa=$ $1 / k_{F} a$ (circles). The solid lines for negative and positive $\kappa$ represent the limiting behavior of the BCS theory (with the Gorkov-Melik-Barkhudarov correction) and the ideal BEC, respectively. For reference, we also plot nonuniversal results for hard-sphere (triangles) and soft-sphere (squares) bosons (Ref. [23]). scale $l_{0}$ such that

$$
\varepsilon_{\mathbf{k}}= \begin{cases}k^{2} / 2 m, & k<2 \pi / l_{0} \\ \infty, & k>2 \pi / l_{0}\end{cases}
$$

yielding

$$
m / 4 \pi a=U^{-1}-U_{*}^{-1}, \quad U_{*}=-\pi l_{0} / m .
$$

It is straightforward to generalize the DDMC method for resonant fermions [14] to the continuous model (1). One starts by expanding the partition function $Z=T r e^{-\beta H}$, where $\beta=1 / k_{B} T$, in powers of $U$. The resulting Feynman diagrams consist of four-point interaction vertices connected by free single-particle propagators $G_{\sigma}^{(0)}$. A diagram of a given order $p$ is described by the space-time configuration of the vertices $\left.\mathcal{S}_{p}=\left\{\left(\mathbf{x}_{j}, \tau_{j}\right), j=1, \ldots, p\right)\right\}$ $(\tau \in[0, \beta]$ is the imaginary time) and the topology of propagator lines connecting them without integration over the vertex positions-the latter is done by the Monte Carlo sampling process. Next, one observes [19] that the sum over all topologies is given by $\operatorname{det} \mathbf{A}^{\uparrow}\left(\mathcal{S}_{p}\right) \times$ $\operatorname{det} \mathbf{A}^{\downarrow}\left(\mathcal{S}_{p}\right)$, where $\mathbf{A}^{\sigma}$ is the $p \times p$ matrix, $A_{i j}^{\sigma}\left(\mathcal{S}_{p}\right)=$ $G_{\sigma}^{(0)}\left(\mathbf{x}_{i}-\mathbf{x}_{j}, \tau_{i}-\tau_{j}\right)$. In the case of equal densities of the spin components, the weight of a configuration $\mathcal{S}_{p}$ is positive definite:

$$
d \mathcal{P}\left(p, \mathcal{S}_{p}\right)=(-U)^{p}\left|\operatorname{det} \mathbf{A}\left(\mathcal{S}_{p}\right)\right|^{2} \prod_{j=1}^{p} d \tau_{j} d \mathbf{x}_{j} .
$$

The partition function $Z=\sum_{p=0}^{\infty} \int_{\mathcal{S}_{p}} d \mathcal{P}$ is calculated stochastically according to the standard Metropolis-Rosenbluth ${ }^{2}$-Teller ${ }^{2}$ algorithm ensuring that configurations $\mathcal{S}_{p}$ are generated with the probability density given by Eq. (5). The Monte Carlo updates are based on a worm algorithm for the four-point correlation function [15] $G_{2}\left(\mathbf{x}, \tau ; \mathbf{x}^{\prime}, \tau^{\prime}\right)=\left\langle\mathcal{T}_{\tau} P(\mathbf{x}, \tau) P^{\dagger}\left(\mathbf{x}^{\prime}, \tau^{\prime}\right)\right\rangle$, where $\mathcal{T}_{\tau}$ indicates time ordering, $P(\mathbf{x}, \tau)=\Psi_{\uparrow}(\mathbf{x}, \tau) \Psi_{\downarrow}(\mathbf{x}, \tau)$ is the pair annihilation operator, and $\langle\cdots\rangle$ is the thermal average. The asymptotic value of $\iint d \tau d \tau^{\prime} G_{2}\left(\mathbf{x}, \tau ; \mathbf{x}^{\prime}, \tau^{\prime}\right)$ as $\left|\mathbf{x}-\mathbf{x}^{\prime}\right| \rightarrow \infty$ is proportional to the condensate density.

Up to statistical errors, the DDMC calculations yield exact results for a finite system-in our case a cubic box of a linear size $L$ with periodic boundary conditions. An efficient way of finding $T_{c}$ in the thermodynamic limit $L \rightarrow$ $\infty$ is to employ the technique of Binder crossings [20] for $R=L^{1+\eta} \int d \mathbf{x} d \mathbf{x}^{\prime} d \tau d \tau^{\prime} G_{2}\left(\mathbf{x}, \tau ; \mathbf{x}^{\prime}, \tau^{\prime}\right) /\left(\beta L^{3}\right)^{2} \quad$ [where $\eta \approx 0.038$ for the 3D $U(1)$ universality class], as discussed in detail in Ref. [15]. It is expected that at the critical point $R$ becomes scale invariant. By analyzing the crossings of the family of $R(L, \beta)$ curves one can obtain $T_{c}$ with an accuracy of a fraction of percent with a relatively small number of particles. The thermodynamic limit of the number density is obtained from a linear extrapolation of $n(L)$ as a function of $1 / L$. An example of the finite-size analysis for a typical set of parameters is given in Fig. 2. 


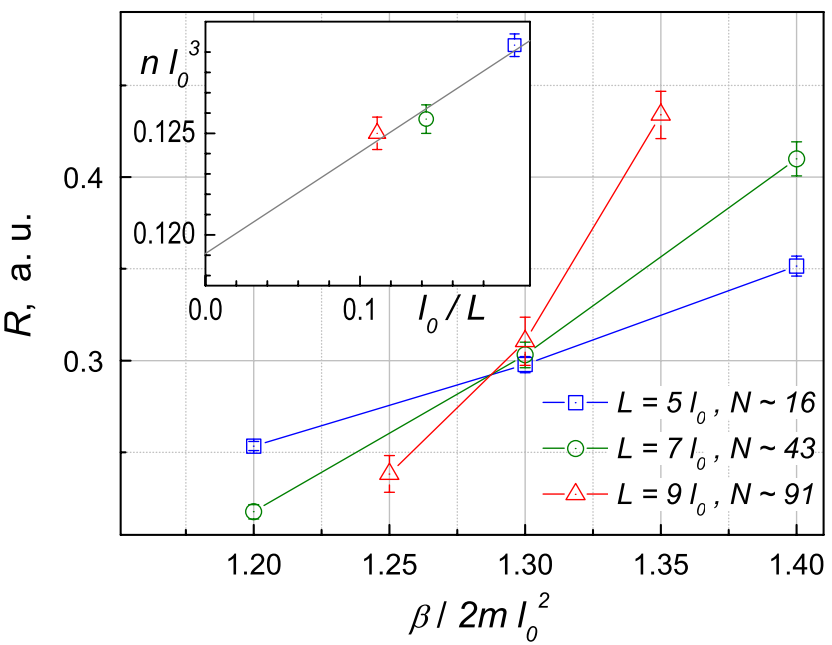

FIG. 2 (color online). Finite-size analysis for $c=0.83, \mu=$ 0.36 , corresponding to $U \approx-7.519$ and $a \approx 1.52$ (in the units of $\left.m=1 / 2, l_{0}=1\right)$ yielding $\beta_{c}=1.290(8)$. The error bars are 1 standard deviation and were calculated using the blocking method. Inset: The thermodynamic limit value of the number density is obtained via a linear fit of $n(L)$ vs $1 / L$. In this case, $n l_{0}^{3}=0.119(2)$, which results in $\zeta=0.492(3), T_{c}^{(\zeta)} / \varepsilon_{F}^{(\zeta)}=$ $0.335(6)$, and $\kappa=0.432(3)$.

In order to obtain the universal answer for $T_{c} / \varepsilon_{F}$ one finally has to take the limit of $\zeta=n^{1 / 3} l_{0} \rightarrow 0$ by extrapolating numerical data for $T_{c}^{(\zeta)} / \varepsilon_{F}^{(\zeta)}$ to the dilute limit. We keep $l_{0}$ constant and take the limit by lowering the chemical potential $\mu$ and diluting the system. One can show [15] that the leading-order corrections should be linear in $\zeta \ll$ 1: $T_{c}^{(\zeta)} / \varepsilon_{F}^{(\zeta)}=T_{c} / \varepsilon_{F}+$ const $\times \zeta+o(\zeta)$. The calculation strategy is as follows: at unitarity $(\kappa \equiv 0)$, we fix $U=U_{*}$ according to Eq. (4) and perform a series of simulations for different values of $\mu$, yielding a set of $T_{c}^{(\zeta)} / \varepsilon_{F}^{(\zeta)}$. Then, the universal value of the critical temperature follows from the linear extrapolation of $T_{c}^{(\zeta)} / \varepsilon_{F}^{(\zeta)}$ to $\zeta \rightarrow 0$.

To obtain $T_{c} / \varepsilon_{F}$ away from the resonance, the procedure has to be modified. Taking the dilute limit for each value of $\kappa \neq 0$ requires that $a \rightarrow \infty$ in such a way that $1 / k_{F} a$ tends to a fixed finite value $\kappa$. We note that the universal value of the chemical potential obeys $\mu\left(T_{c}\right) / \varepsilon_{F} \equiv 2 m g(\kappa)$, with some function $g(\kappa)$, or, equivalently, $\lim _{\zeta \rightarrow 0} \mu^{(\zeta)}\left(T_{c}\right) a^{2}=$ $g(\kappa) / \kappa^{2}$ implying that for each $\kappa$ one has to keep $\mu a^{2}=$ const. Substituting $a^{2}=\mu / c$ into (4) gives

$$
U=U_{*}\left(1 \pm \frac{m U_{*}}{4 \pi} \sqrt{\frac{\mu}{c}}\right)^{-1},
$$

where the upper (lower) sign corresponds to the BEC side $a>0$ (BCS side $a<0$ ). We thus pick a value of $c$ and perform a series of simulations for smaller and smaller values of $\mu$ with $U$ from Eq. (6). Each simulation yields a finite- $\zeta$ estimate for the critical temperature $T_{c}^{(\zeta)}(c)$, density $n^{(\zeta)}(c)$, and $\kappa^{(\zeta)}(c)$. After linear extrapolations to $\zeta \rightarrow$
0 we determine the physical value of $T_{c} / \varepsilon_{F}$ and the corresponding value of $\kappa$.

In Fig. 3 we show results for the critical temperature as a function of $\zeta$. For comparison and consistency analysis of $T_{c}$ at unitarity, we also plot the data for the Hubbard model [14] as a function of the filling factor $\nu$, which plays the same role as $\zeta$ in the present model. Note that the nonuniversal corrections to $T_{c} / \varepsilon_{F}$ in $\zeta$ turn out to be positive and much smaller (at unitarity) than for the Hubbard model. The former fact is important for the simulation efficiency, since the computational complexity of the DDMC technique scales as $(\beta U N)^{3}$, where $N$ is the number of fermions, and it is advantageous to simulate at higher temperatures.

It is important to note that at high densities the $T_{c}^{(\zeta)} / \varepsilon_{F}^{(\zeta)}$ curves are almost constant and the true asymptotic low- $\zeta$ behavior develops only below $\zeta \approx 0.75$. For a reliable extrapolation it is crucial to vary the density by at least an order of magnitude, and we did so by diluting the system down to $n \approx 0.04 / l_{0}^{3}(\zeta \approx 0.35)$, where we were limited by the low values of the absolute critical temperature itself. Unfortunately, no dilute-limit extrapolation was performed in Ref. [16] (their value for $\zeta \approx 0.93$ is shown by the diamond in Fig. 3). The total simulation time required to obtain this set of data was approximately $10^{6} \mathrm{CPU}$ hours on Opteron-class workstations.

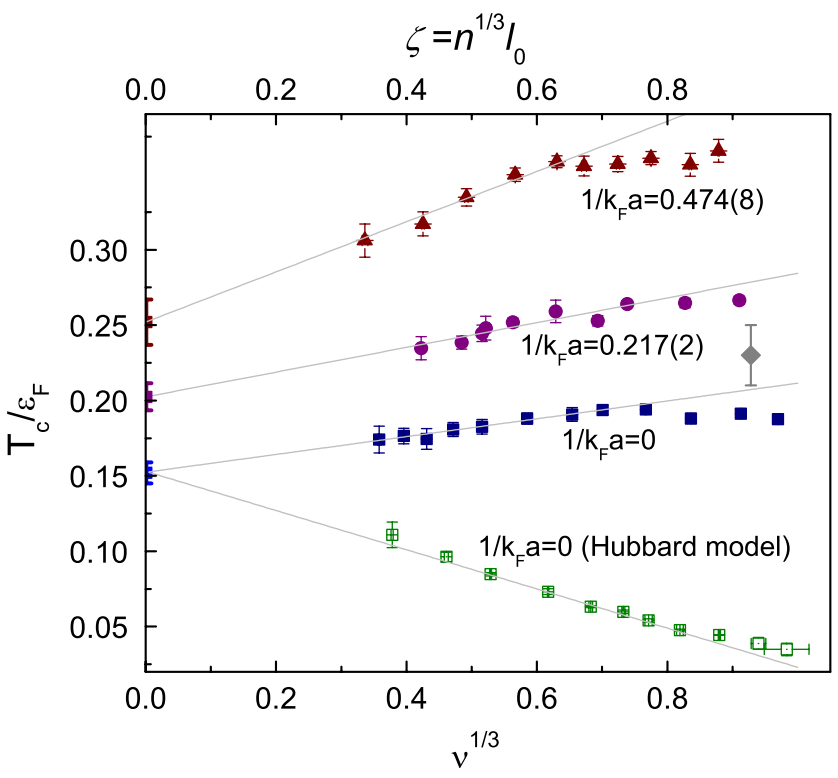

FIG. 3 (color online). The extrapolation of the simulation results to the universal limit $\zeta=n^{1 / 3} l_{0} \rightarrow 0$. The procedure yields $T_{c} / \varepsilon_{F}=0.152(9), 0.202(9)$, and $0.252(15)$ for $\kappa=$ $1 / k_{F} a=0$ (squares), 0.217(2) (circles), and 0.474(8) (triangles), correspondingly. For comparison, we also plot our results for the Hubbard model (open squares) adapted from Ref. [14]. The estimate of Ref. [16] at $\kappa=0$ (obtained for finite $\zeta \approx 0.93$ ) is shown by the diamond. Solid lines are linear fits. 
At unitarity, the extrapolated result for $T_{c}^{(\zeta)} / \varepsilon_{F}^{(\zeta)}$ yields an answer which is in perfect agreement with $T_{c} / \varepsilon_{F}=$ 0.152 (7) obtained independently from the $\nu \rightarrow 0$ extrapolation of the Hubbard model data [14]. In the latter case, the universal value is approached from below (see Fig. 3). This agreement unambiguously demonstrates that our treatment of nonuniversal corrections is reliable in the simulated parameter range (linear fits for $\zeta<0.75$ ). Away from unitarity we find $T_{c} / \varepsilon_{F}=0.202(9)$ and $0.252(15)$ for $\kappa=$ $0.217(2)$ and $0.474(8)$, respectively.

The results for the strongly correlated regime essentially determine the general shape of the universal curve $\left[T_{c} / \varepsilon_{F}\right](\kappa)$ shown in Fig. 1. Deep in the BEC regime $(\kappa \gg 1)$ the critical temperature is that of a weakly interacting Bose gas of strongly bound dimers which is expected to increase on approach to the resonance. In the BCS limit $(\kappa<0,|\kappa| \gg 1)$ the $T_{c}$ curve starts from exponentially small values for $\kappa \rightarrow-\infty$, and thus the crossover between the two limiting regimes necessarily features a maximum in $T_{c} / \varepsilon_{F}$. The results in Fig. 1 clearly show that this maximum must be on the BEC side $(\kappa>0)$. The value at the maximum appears to be surprisingly high. For comparison, we show in Fig. 1 the critical temperatures of a Bose gas with hard- and soft-core sphere potentials with scattering length $a_{B}=0.6 a$. The tremendous computational cost required to determine each point in the crossover regime reliably did not allow us to precisely locate the position of the maximum in the $k_{F} a \sim 1$ region.

The behavior of the critical temperature on the BEC side revealed by our simulations suggests that the short-range structure of the strongly correlated state is radically different from that of the compact-molecule Bose gas (and, obviously, also from that of the BCS state) in a broad range of $\kappa$. In other words, we are dealing with two crossoversone is from BCS to the substantial unitarity regime and the other is from the unitarity regime to BEC.

To summarize, we performed first-principle simulations of the two-component unpolarized Fermi gas with resonant interparticle interaction obtaining the universal critical temperature $T_{c} / \varepsilon_{F}=0.152(7)$ at the unitarity point $\kappa=$ $1 / k_{F} a=0$ thereby resolving the earlier controversy between the results of Refs. [15] and [16]. We also obtain $T_{c}$ away from unitarity on the BEC side allowing one to sketch the general dependence $\left[T_{c} / \varepsilon_{F}\right](\kappa)$ with a maximum on the BEC side, in a good quantitative agreement with the mean-field-type prediction by Haussmann et al. [8]. After our results were announced [21], the Seattle group reconsidered their previous estimate of the critical temperature [22]. The new results are in excellent agreement with the values claimed here both at and away from unitarity.

The simulations were performed on the supercomputers Hreidar at ETH Zurich, Mammoth at the University of
Sherbrooke, Typhon and Athena at the College of Staten Island, CUNY. The work was supported by the National Science Foundation under Grant No. PHY-0653183. E. B. was partially supported by IFRAF.

[1] See, e.g., S. Giorgini, L. P. Pitaevskii, and S. Stringari, arXiv:0706.3360 [Rev. Mod. Phys. (to be published)], and references therein.

[2] H. Heiselberg and M. Hjorth-Jensen, Phys. Rep. 328, 237 (2000).

[3] A. Gezerlis and J. Carlson, Phys. Rev. C 77, 032801(R) (2008), and references therein.

[4] F. X. Bronold and H. Fehske, Phys. Rev. B 74, 165107 (2006).

[5] T.-L. Ho, Phys. Rev. Lett. 92, 090402 (2004).

[6] D. M. Eagles, Phys. Rev. 186, 456 (1969); A. J. Legett, in Modern Trends in the Theory of Condensed Matter, edited by A. Pekalski and R. Przystawa (Springer-Verlag, Berlin, 1980).

[7] P. Noziéres and S. Schmitt-Rink, J. Low Temp. Phys. 59, 195 (1985); M. Randeria, in Bose-Einstein Condensation, edited by A. Griffin et al. (Cambridge University Press, Cambridge, England, 1995).

[8] R. Haussmann, W. Rantner, S. Cerrito, and W. Zwerger, Phys. Rev. A 75, 023610 (2007).

[9] Y. Nishida, Phys. Rev. A 75, 063618 (2007).

[10] P. Nikolić and S. Sachdev, Phys. Rev. A 75, 033608 (2007).

[11] M. Y. Veillette, D. E. Sheehy, and L. Radzihovsky, Phys. Rev. A 75, 043614 (2007).

[12] V. K. Akkineni, D. M. Ceperley, and N. Trivedi, Phys. Rev. B 76, 165116 (2007).

[13] N. Barnea, arXiv:0803.1349.

[14] E. Burovski, N. Prokof'ev, B. Svistunov, and M. Troyer, Phys. Rev. Lett. 96, 160402 (2006).

[15] E. Burovski, N. Prokof'ev, B. Svistunov, and M. Troyer, New J. Phys. 8, 153 (2006).

[16] A. Bulgac, J.E. Drut, and P. Magierski, Phys. Rev. Lett. 96, 090404 (2006).

[17] D. J. Scalapino and R. L. Sugar, Phys. Rev. Lett. 46, 519 (1981); R. Blankenbecler, D. J. Scalapino, and R. L. Sugar, Phys. Rev. D 24, 2278 (1981).

[18] E. M. Lifshitz and L. P. Pitaevskii, Statistical Mechanics, Part 2 (Pergamon, New York, 1980).

[19] A. N. Rubtsov, V. V. Savkin, and A. I. Lichtenstein, Phys. Rev. B 72, 035122 (2005).

[20] K. Binder, Phys. Rev. Lett. 47, 693 (1981).

[21] See, e.g., http://online.itp.ucsb.edu/online/coldatoms07/ svistunov

[22] A. Bulgac, J. E. Drut, and P. Magierski, arXiv:0803.3238. Since the lowest density reported was about $n \geqslant 0.7 / l_{0}^{3}$, we can only speculate that an additional lattice discretization present in the model somehow helps in reducing the nonuniversal corrections.

[23] S. Pilati, S. Giorgini, and N. Prokof'ev, Phys. Rev. Lett. 100, 140405 (2008). 